\title{
Novel Concept of Attaching Endoscope Holder to Microscope for Two Handed Endoscopic Tympanoplasty
}

\author{
Mubarak M. Khan ${ }^{1} \cdot$ Sapna R. Parab ${ }^{1}$
}

Received: 6 July 2015 / Accepted: 17 September 2015/Published online: 6 November 2015

(C) Association of Otolaryngologists of India 2015

\begin{abstract}
The well established techniques in tympanoplasty are routinely performed with operating microscopes for many decades now. Endoscopic ear surgeries provide minimally invasive approach to the middle ear and evolving new science in the field of otology. The disadvantage of endoscopic ear surgeries is that it is one-handed surgical technique as the non-dominant left hand of the surgeon is utilized for holding and manipulating the endoscope. This necessitated the need for development of the endoscope holder which would allow both hands of surgeon to be free for surgical manipulation and also allow alternate use of microscope during tympanoplasty. To report the preliminary utility of our designed and developed endoscope holder attachment gripping to microscope for two handed technique of endoscopic tympanoplasty. Prospective Non Randomized Clinical Study. Our endoscope holder attachment for microscope was designed and developed to aid in endoscopic ear surgery and to overcome the disadvantage of single handed endoscopic surgery. It was tested for endoscopic Tympanoplasty. The design of the endoscope holder attachment is described in detail along with its manipulation and manoeuvreing. A total of 78 endoholder assisted type 1 endoscopic cartilage tympanoplasties were operated to evaluate its feasibility for the two handed technique and to evaluate the results of endoscopic type 1 cartilage tympanoplasty. In early follow
\end{abstract}

Electronic supplementary material The online version of this article (doi:10.1007/s12070-015-0916-6) contains supplementary material, which is available to authorized users.

Mubarak M. Khan

drmubarakkhan@yahoo.co.in; ent.khan@gmail.com

1 Department of Otorhinolaryngology, M.I.M.E.R. Medical College, Pune 410507, India up period ranging from 6 to 20 months, the graft uptake was seen in 76 ears with one residual perforation and 1 recurrent perforations giving a success rate of $97.435 \%$. Our endocsope holder attachment for gripping microscope is a good option for two handed technique in endoscopic type 1 cartilage tympanoplasty. The study reports the successful application and use of our endoscope holder attachment for gripping microscope in two handed technique of endoscopic type 1 cartilage tympanoplasty and comparable results with microscopic techniques.

Level of evidence: IV.

Keywords Justtach · Endoscope holder . Endoscopic cartilage tympanoplasty

\section{Introduction}

Endoscopic surgeries have gained popularity not only in Otolaryngology but also in other surgical fields. Endoscopes and the Hopkins rod lens system have revolutionized the medical field in last half century. Endoscopic approach for paranasal sinuses is well known. The well established techniques in tympanoplasty are routinely performed with operating microscopes for many decades now. Endoscopic ear surgeries provide minimally invasive approach to the middle ear and evolving new science in the field of otology which is in its nascent stage. Migrating from classical Wullstein's microscopic tympanoplasty techniques to endoscopic tympanoplasty techniques is slow and tough but still a new horizon rising in the field of otology. Rigid endoscopes have been used by otorhinolaryngologists for sinus surgery and recently even for otologic surgeries. The advantages of endoscopic sinus surgeries are well known. In otologic surgery too, 


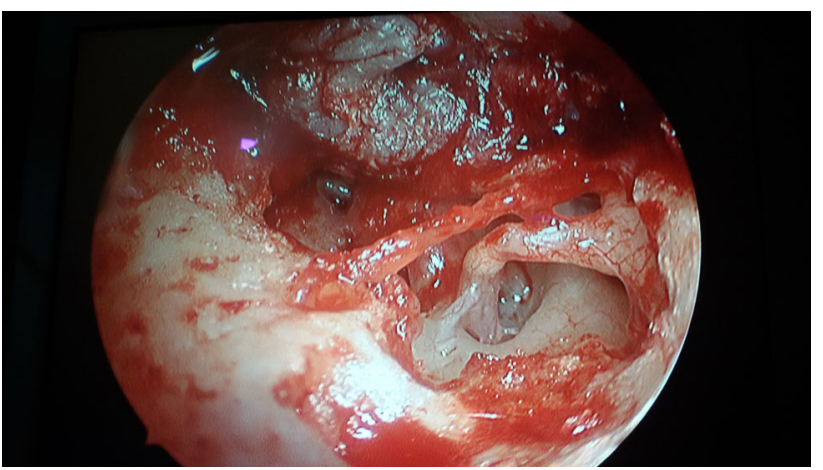

Fig. 1 Endoscopic view of middle ear showing inaccecible areas by microscope

endoscopes have several advantages to offer. (1) It allows visualization of the whole tympanic membrane and the ear canal without having to manipulate the patient's head or the microscope, (2) it extends the operative field in transcanal procedures into structures usually hidden from the microscope (anterior tympanic perforation, posterior retraction pocket, facial recess, and hypotympanum) (Fig. 1), (3) it visualizes structures from multiple angles as opposed to the microscope's single axis along the ear canal [1]. Disadvantage of the endoscopic surgeries is that it is one-handed surgical technique as the non-dominant hand of the surgeon is utilized for holding the endoscope. During the haemorrhage and drilling, it becomes difficult to use suction and instruments both along with endoscope in single handed technique. Even during fogging, one needs to remove endoscope again and again for lense cleaning. As endoscopic ear surgery (EES) is in developing stage, many of the times during drilling one needs to use microscope. And whenever one wants that multiple magnifications to visualize middle ear structures sometimes during ontological surgeries, microscope may be needed. This necessitated the need for development of the endoscope holder attachment which would allow both hands of the surgeon to be free for surgical manipulation during EES and allow alternate use of microscope whenever needed during tympanoplsty. The purpose of this study was to report the early and preliminary experience with the endoscope holder attachment for gripping microscope for endoscopic cartilage tympanoplasty. Incorporating the endoscope holder attachment into the armamentarium of Otorhinolaryngologic surgeries will not only contribute to the concept of documentation but will also augment the expanding uses of endoscope both for diagnostic and therapeutic use. The use of endoscope holder attachment in otolaryngologic surgeries may be considered as a new horizon. The idea is not just to hold the endoscope during surgeries, but to move and manipulate it as the left hand does it more or less. Operating Microscopes have range of motion in space during ontological surgeries for focusing the desired object for magnification. This whole range of motions can be applied for driving the endoscope smoothly in any biological cavities once an endoscope holding attachment is fixed to optical system of any operating microscope (Patent Application No. 3300-Mum-2013): [2]. Robotic arms can do this, but they are too expensive. Hence this is an economical way to simulate it. Every otologist is well versed with the use of the microscope for ear surgeries. Operating microscope can be either mounted on floor stand, or ceiling or can be fitted on to the operating table in portable version. These operating microscopes have 360 degree rotation and motion in space for focusing and magnification of object. The same motions can be helpful for driving the endoscope in biological cavities. This is achieved by holding endoscope with metallic plate and gripping the optical system of any microscope just above objective lense (Patent Application No. 3300-Mum2013): [2].

\section{Materials and Methods}

Prior to developing the endoscope holder attachment, we performed the single handed technique of EES for 5 years. However due to the technical difficulties of single handed surgery, the Endoholder attachment was devised.

A total of 78 consecutive primary endoscopic type 1cartilage tympanoplasties for pars tensa perforations were operated with our endoscope holder attachment gripping to microscope (Fig. 2). from September 2013 to November 2014 in M.I.M.E.R Medical College and Sushrut ENT Hospital with a follow up period ranging from 6 to 20 months. There was no ossicular chain impairment. There were 43 males and 33 females in the study group. The mean age of the study group was $28.75 \pm 4.46$ years. The youngest patient was 11 years of age and the oldest was 47 years of age. The average preoperative Air Bone Gap in the study group was $33.25 \pm 2.74 \mathrm{~dB}$ and post operative $\mathrm{AB}$ gap closed up to $8.57 \pm 2.24$.

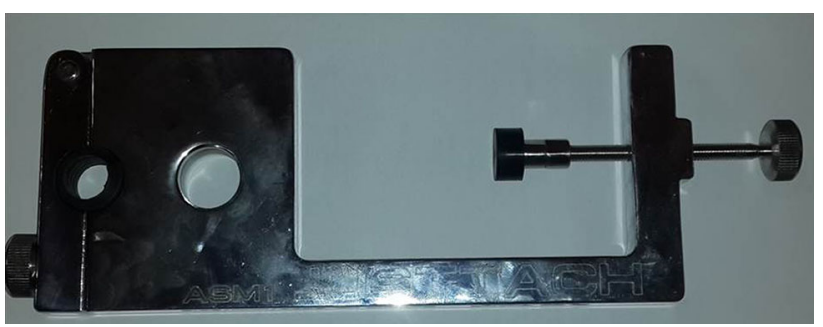

Fig. 2 Endoscope holder with Microscope gripping attachment (Justtach) 
Design of Our Endoscope Holder Attachment to Microscope (Patent Application No. 3300-Mum-

2013): [2]

The object of the present innovation is to create a device of simple construction, and therefore low cost, which makes it possible

(a) To easily hold any rigid endoscope to any medical operating microscope's optical body (b) To use endoscope and operating microscope alternately during surgery

The innovation achieves this objective by using a piece of a metallic plate of $170 \times 70 \times 12 \mathrm{~mm}$ in dimensions with a circular slot measuring $16 \times 16 \mathrm{~mm}$ in diameter (Fig. 3C) and square slot (Fig. 3A) is rigid endoscopic holder can be affixed to optical system of any operating ENT microscope with 1 tightening screw (Fig. 3B) with the optical observation instrument of the microscope intact.
Fig. 3 Diagrams of endoscope holder with Microscope gripping attachment (Justtach) submitted to patent office

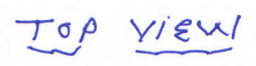

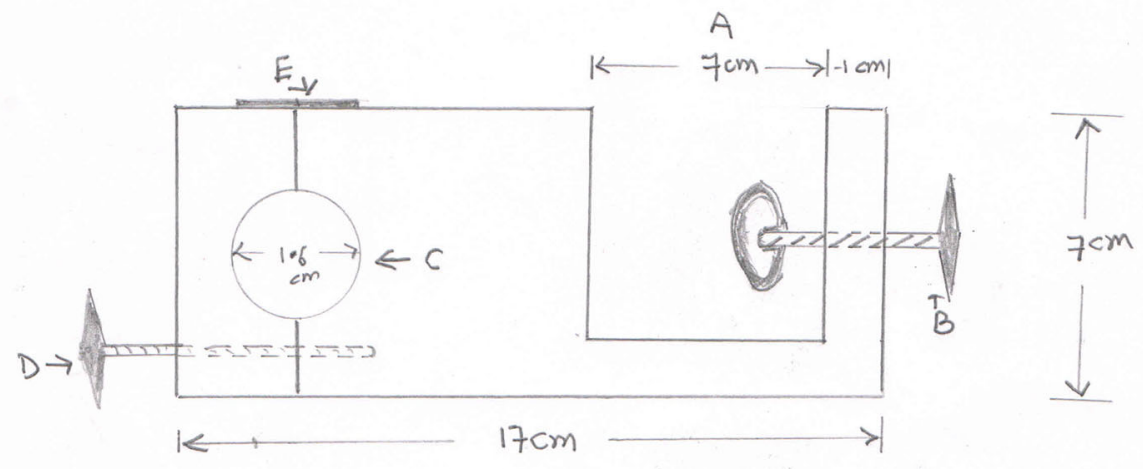

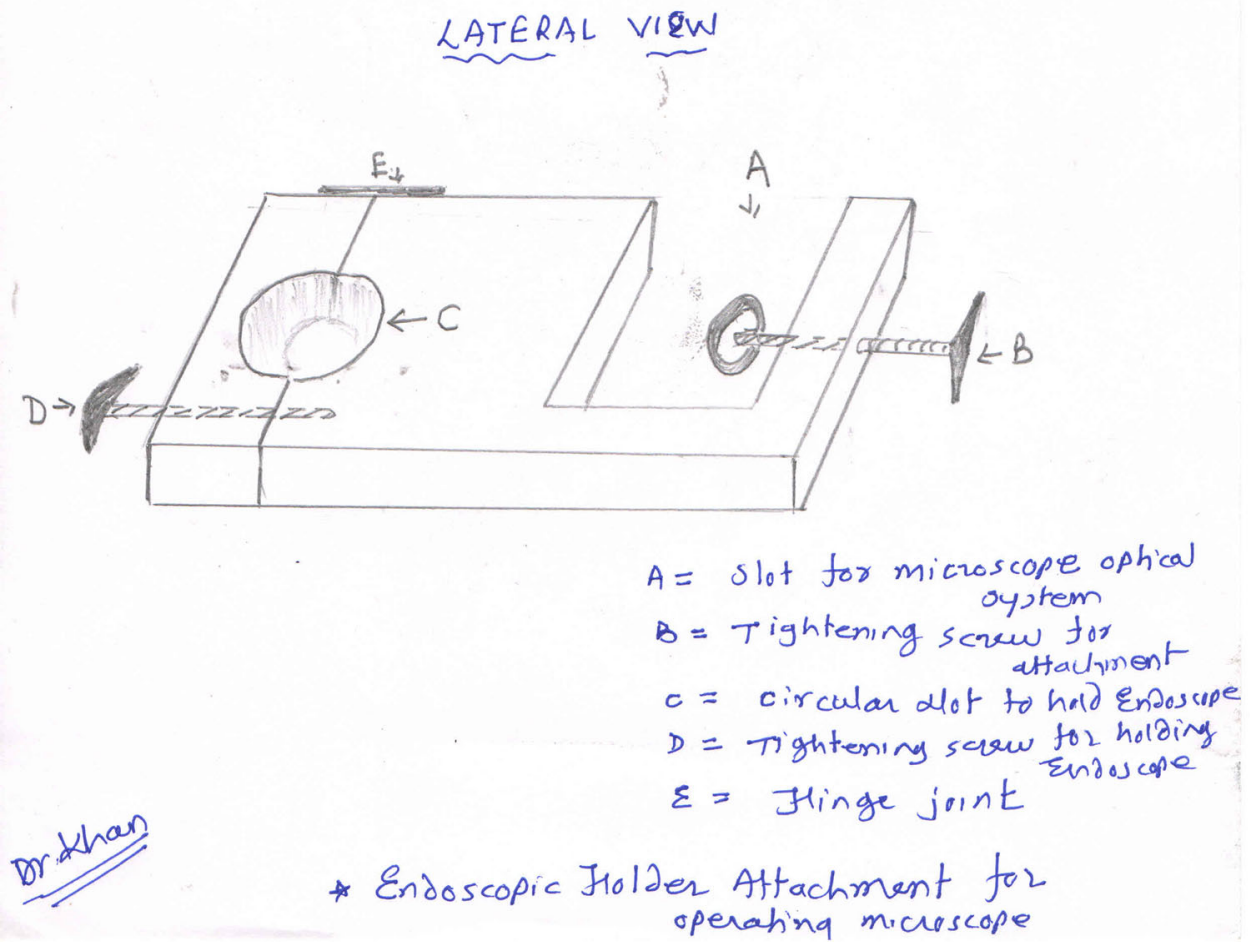


The circular slot (Fig. 3C) of $16 \mathrm{~mm}$ diameter in metallic plate half of which is on main attachment linking to optical system and half of which opens outside to accommodate any rigid endoscope for fixation (action is represented by figures). This opening part of metallic plate is attached to main part with hinge joint (Fig. 3E) with 90 degree of motion. This opening part then can be fixed to main part of metallic plate with adjustable screw (Fig. 3D). In other variant instead of fixing screw (Fig. 3B) on right lateral side, it is on front side. The inner part of circular slot (Fig. 3C) is reinforced with soft silicone or malleable plastic thin material to hold neck of endoscope which holds it firmly and does not cause undue pressure on gentle optical fibers of any rigid endoscope. This slot (Fig. 3C) is at $60 \mathrm{~mm}$ distance from its attached end and can be made of any suitable diameter to accommodate different rigid endoscopes of different diameters.

Thus all actions of operating microscopes are utilized for rigid endoscope which are very valuable when this endoscope holder is directly attached and secured to optical system of any operating microscope.

The described apparatus is combined, in particularly advantageous embodiments, with known operating microscopes and in particular with portable stands, with stands for wall or ceiling attachment, and with stands which have a table base or a floor-clamping device. Combination with a vertically adjustable horizontal arm or with a swivel arm is particularly suitable. The basic idea is to apply all microscope motions in space to drive the endoscope. Our endoscope holder attachment is universal and is compatible with all Endoscopes and cameras of all the brands.

\section{Operating Theatre Requirements}

Zero degree $4 \mathrm{~mm}$ endoscope with triple charge coupled device Camera (Karl Storz, Germany) is held in slot for endoscope on Endoholder attachment and this assembly is firmly fixed to optical system of microscope just above objective lense (Fig. 4). The horizontal arm of microscope stand balanced to avoid undue and uncontrolled vertical motion of microscope. Incorporating the endoscope into the microscope stand with endoscope holder augments the advantages and applicability and allows alternate use of both (Fig. 5).

\section{Preparation of the Patient}

The patient is prepared and draped in the usual fashion with the ipsilateral shoulder pulled down. The ear canal is infiltrated with $2 \%$ lidocaine with 1 in 2,00,000 adrenaline. All patients were operated under general anaesthesia except 5 who were operated under local anaesthesia. Preoperatively, the patient is explained about the endoscopic

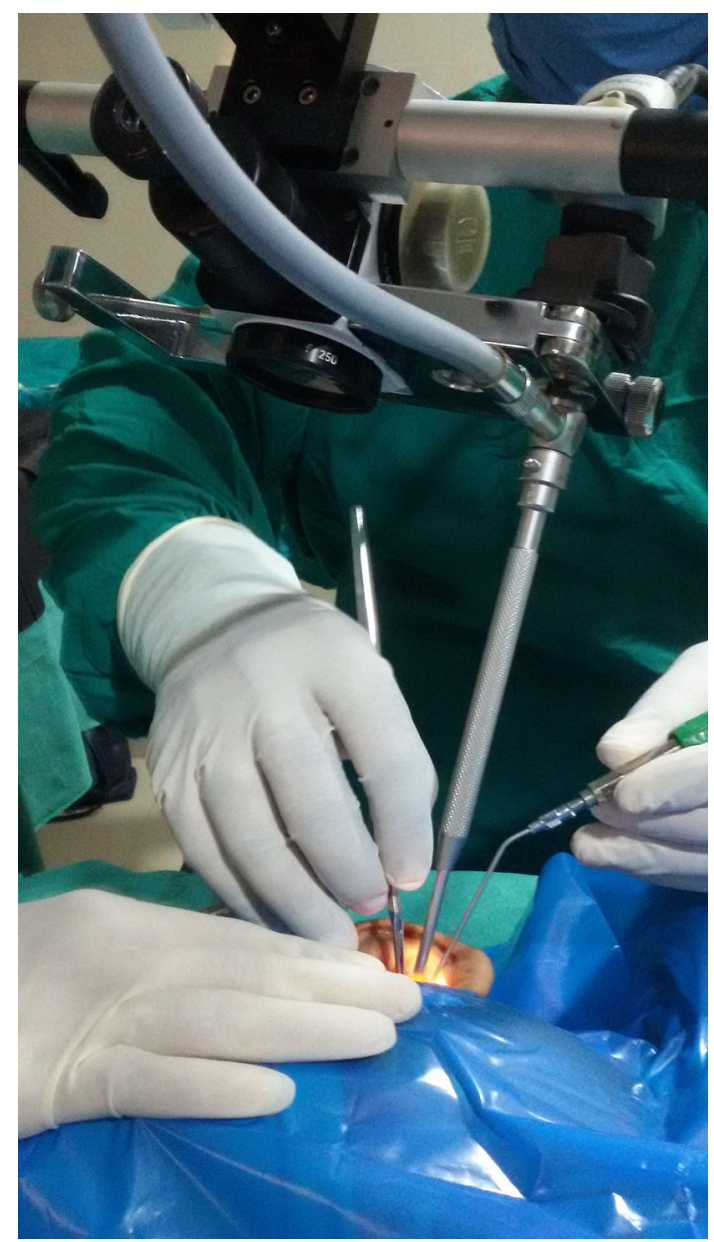

Fig. 4 Endoscope holder with Microscope gripping attachment (Justtach) in use for Endoscopic Tympanoplasty

procedure and is instructed not to move the head in case of local anaesthesia. The assistant steadies the head of the patient by placing the hand over the head of the patient to avoid any accidental head movement and injury in case of local anaesthesia (Fig. 6).

\section{Adapting to the Technique of Two Handed Technique of Endoscopic Tympanoplasty}

After developing the Endoholder attachment to microscope, initially the application and use was demonstrated on Cadaveric temporal bone for endoscopic middle ear dissections (Fig. 7). During the early days of practice of cadaveric temporal bone endoscopic two handed dissection, the manipulations of the endoscope mounted on endoscope holder into and out of the ear canal was practiced. The next step was targeted towards hand eye coordination and the two handed technique of instrumentation (dominant hand for micro ear instruments and non-dominant hand for suctioning). We have dissected five wet 


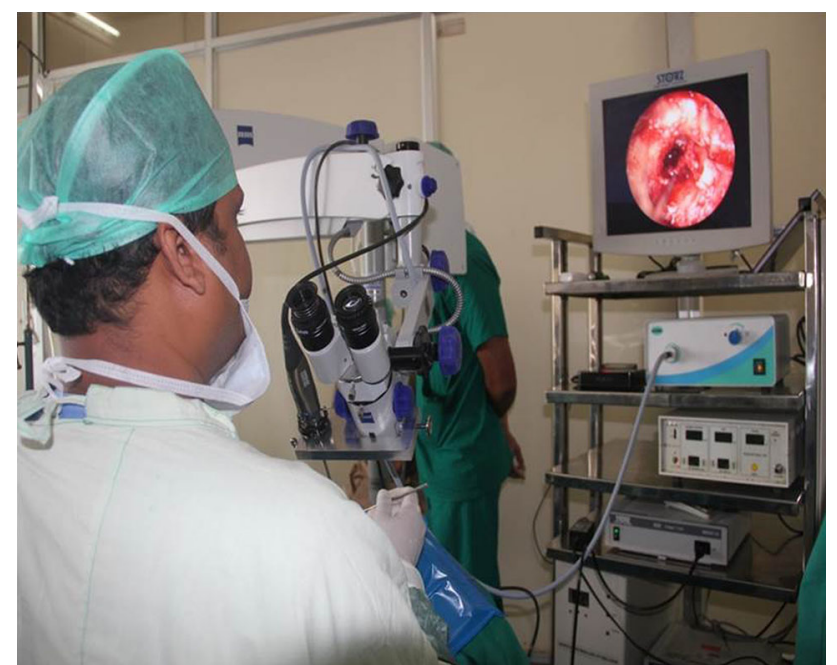

Fig. 5 Endoscope holder with Microscope gripping attachment (Justtach) in use for Endoscopic Tympanoplasty in Operation theatre

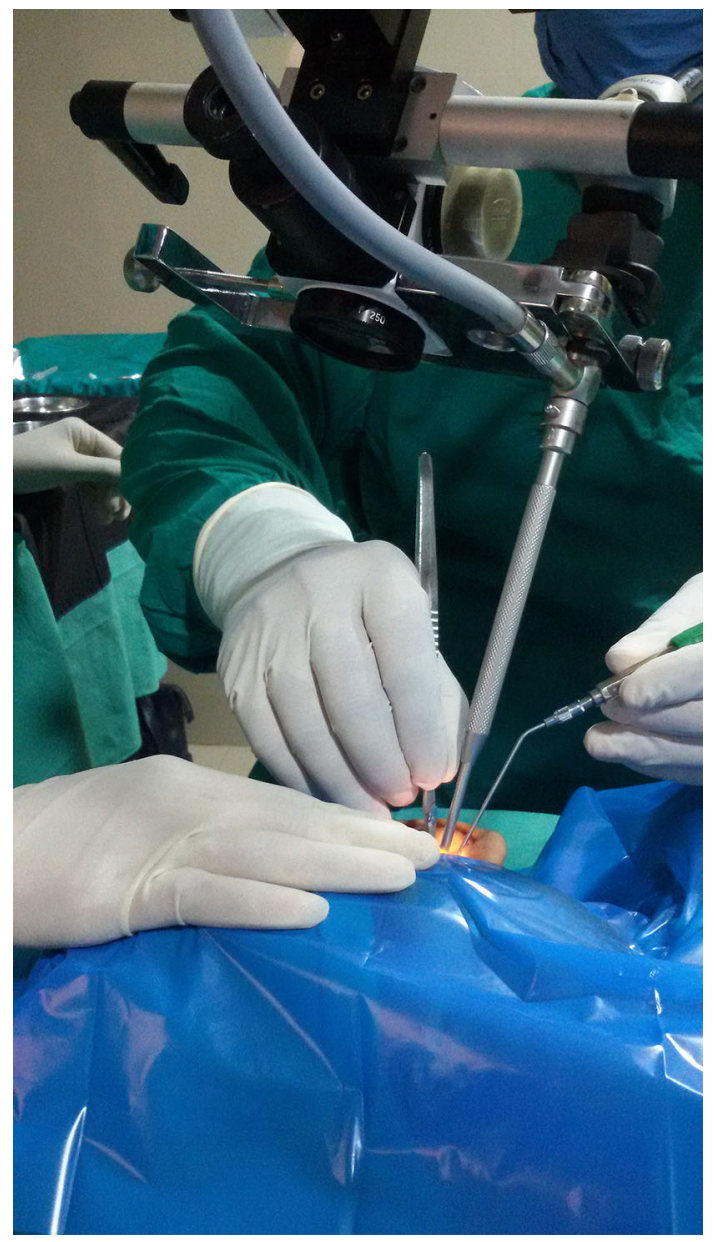

Fig. 6 Endoscope holder with Microscope gripping attachment (Justtach) in use for Endoscopic Tympanoplasty under local anaesthesia

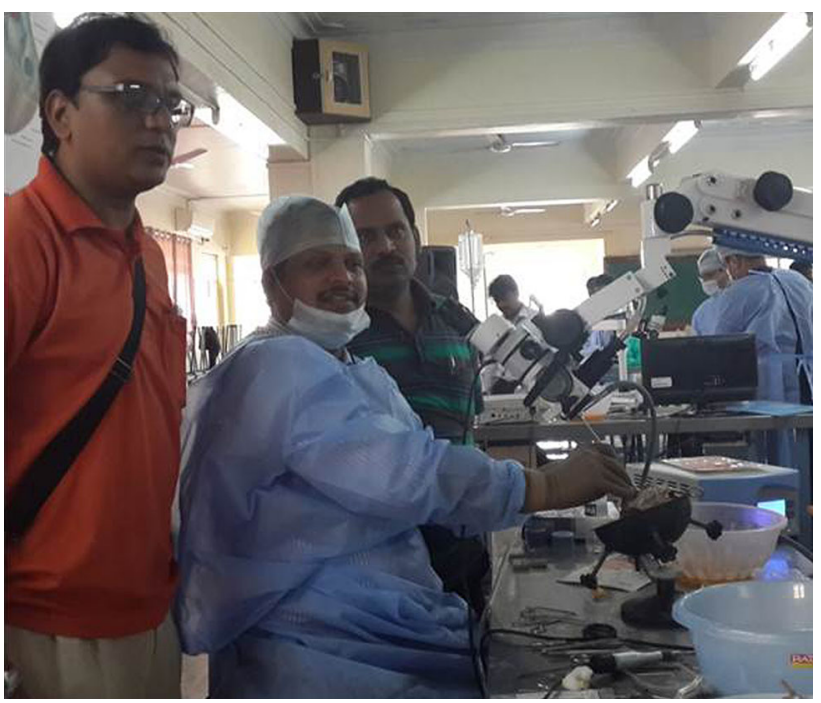

Fig. 7 Endoscope holder with Microscope gripping attachment (Justtach) in use for cadaveric dissection demonstration in courses
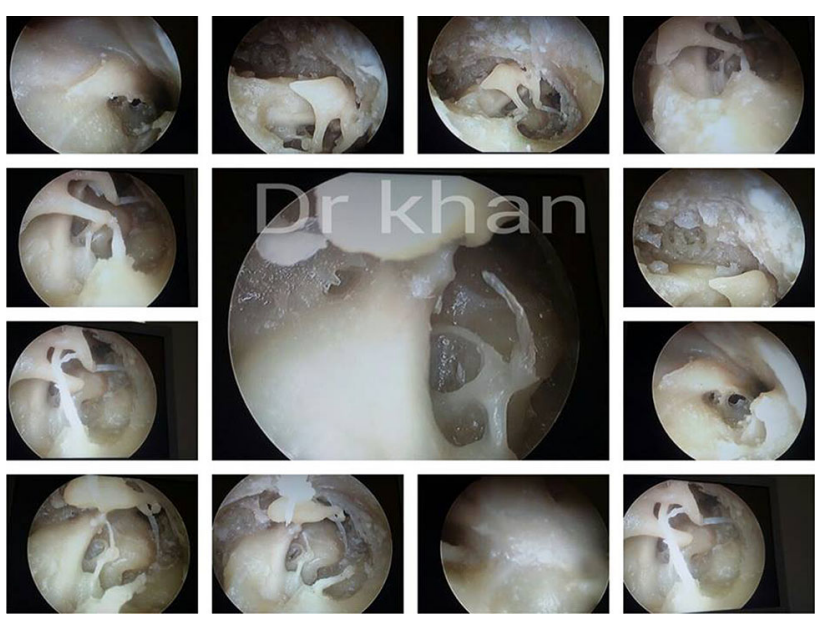

Fig. 8 Cadaveric dissection demonstration. Middle ear Endoscopic anatomy

temporal bones each before performing the EES with the Endoholder attachment (Fig. 8). As the endoscope holder attachment is firmly gripping the Operating Otomicroscope, hence the familiar movement of fine focusing was used for the further smooth advancement of the endoscope into the ear canal. After being thoroughly trained in two handed technique of EES, both the authors started with operating in patients with small perforations and later on to include tympanoplasties for large perforations.

\section{Procedure of Endoscopic Cartilage Tympanoplasty} with Endoscope Holder (Figs. 9, 10, 11, 12, 13, 14, 15, 16)

Instruments routinely used in microscopic ear surgery were used (rosen sickle knife, belluci's knife, picks, elevators, 


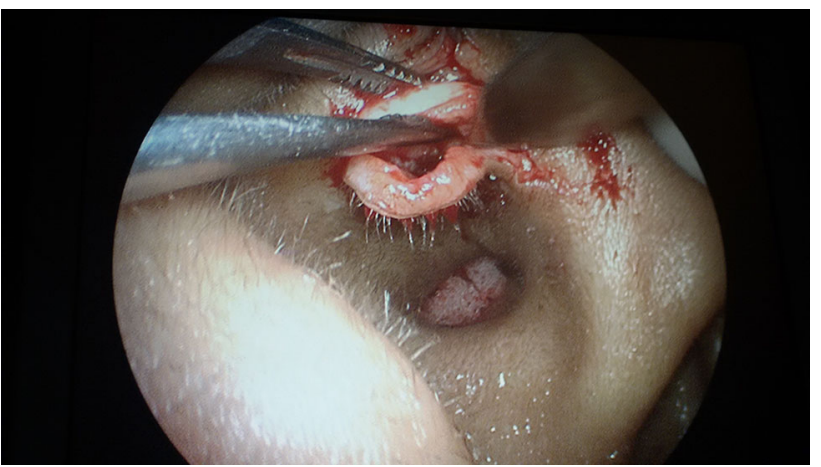

Fig. 9 Tragal cartilage harvestation

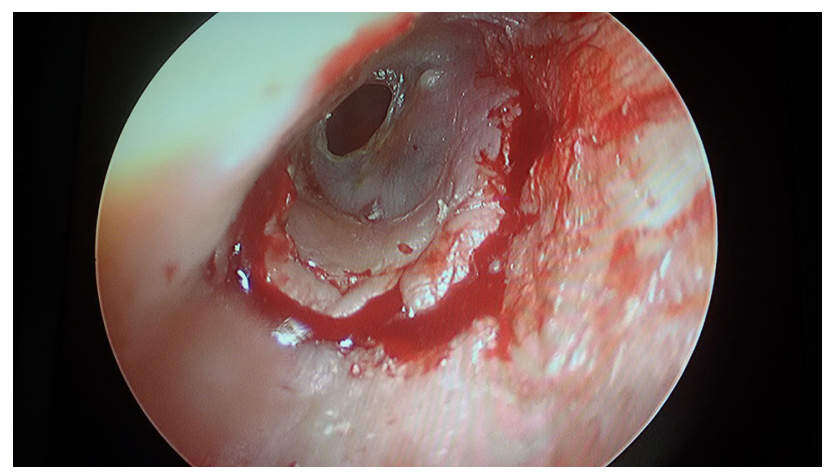

Fig. 10 Left anterior perforation with transcanal incision

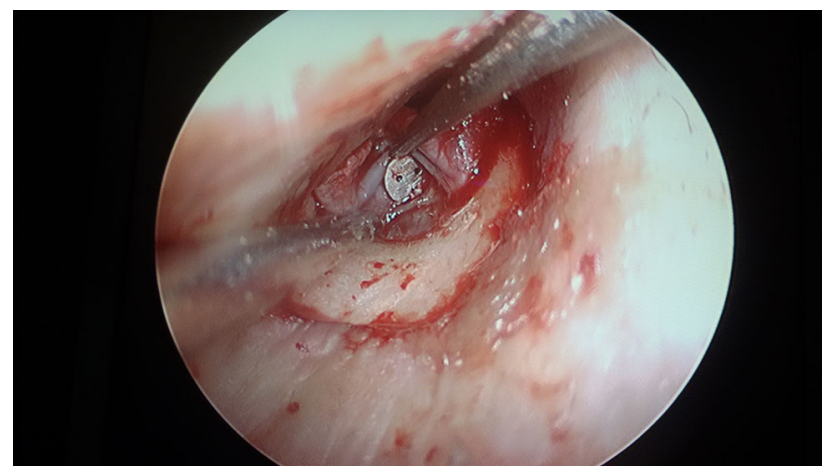

Fig. 11 Elevation of TM flap

and curettes. Using all aseptic precautions, permeatal incision is taken. Tragal cartilage graft is harvested via the horizontal incision on the tragus (Fig. 9). After freshening the edges of the pars tensa perforation, tympanomeatal flap is elevated (Figs. 10,11). Ossicular mobility is confirmed and thorough middle ear inspection done (Fig. 12). The tragal cartilage perichondrium graft is sliced [3-6] with Precise cartilage splitter (Kurz, Germany) to $0.5 \mathrm{~mm}$ thickness with perichondrium retained on one side (Fig. 13). The tragal cartilage is not reinforced by temporalis fascia. Sliced cartilage of $0.5 \mathrm{~mm}$ thickness kept as

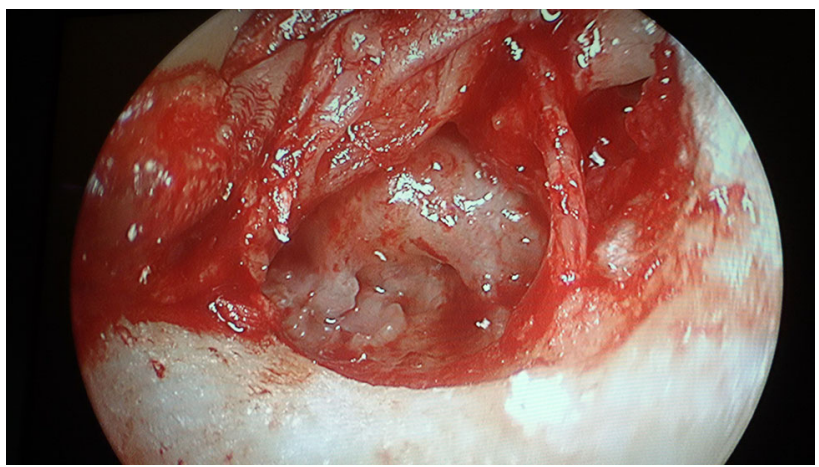

Fig. 12 Endoscopic middle ear view

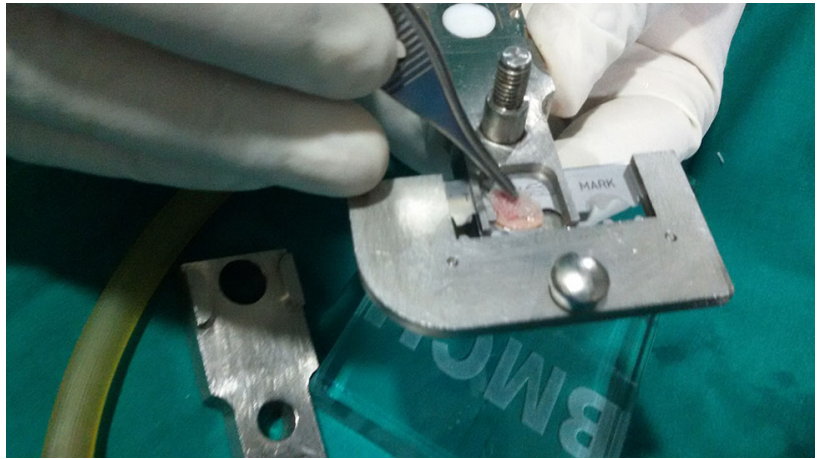

Fig. 13 Tragal cartilage slicing with slicer

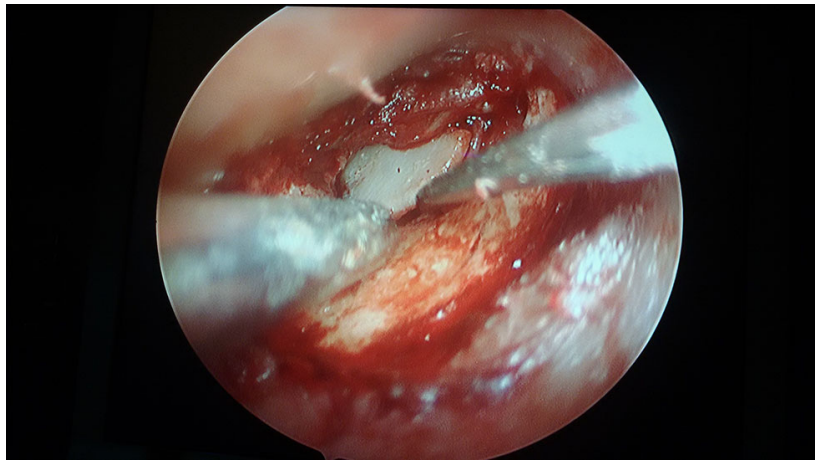

Fig. 14 Sliced tragal cartilage of $0.5 \mathrm{~mm}$ thick shield graft kept as underlay

underlay shield graft (Fig. 14). Tympanomeatal flap reposed back (Fig. 15). Through out during procedure suction is held in left hand to avoid the fogging and to achieve cooling of endoscope generated heat as reported in previous study [16]. Intermittent irrigation is done for cooling of endoscope as well as to achieve cleaning of endoscope lense [16]. This is possible only with two handed endoscopic technique using Endoholder (Fig. 16). The stability rendered to the endoscope with the endoscope holder aids and augments the technical usage of endoscope 


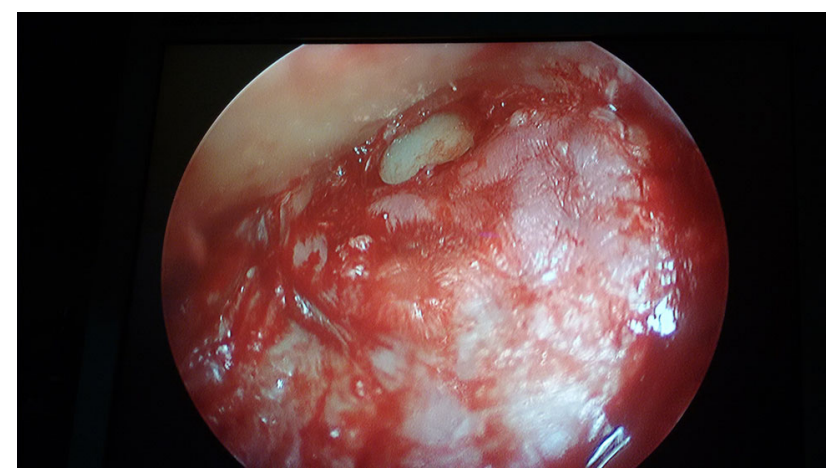

Fig. 15 View after TM flap reposition

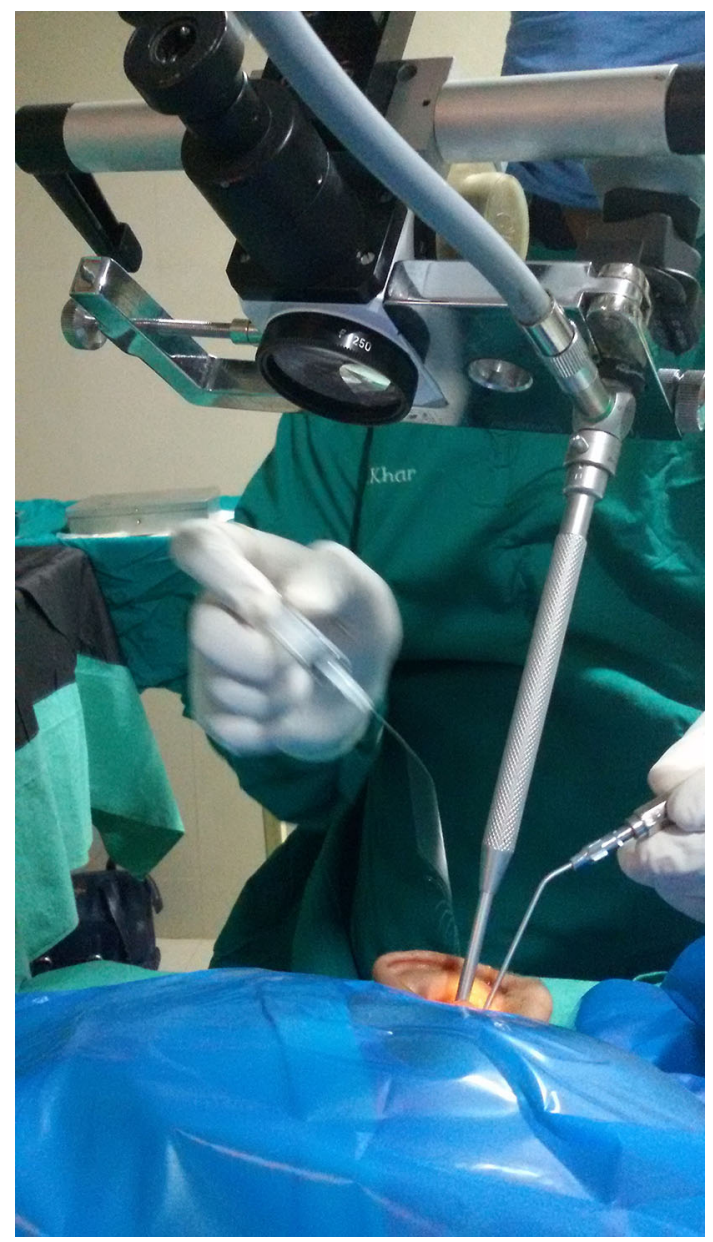

Fig. 16 Simultaneous irrigation and suction during EES for cleaning and cooling the endoscope

in ear surgery during all steps of the routine tympanoplasty (including freshening the edges of perforation, tympanomeatal flap elevation, and underlay technique placement of sliced cartilage graft). No canalplasty is done in this series. Wherever we faced problems of narrow canal, we used specially designed Karl Storz zero degree endoscope

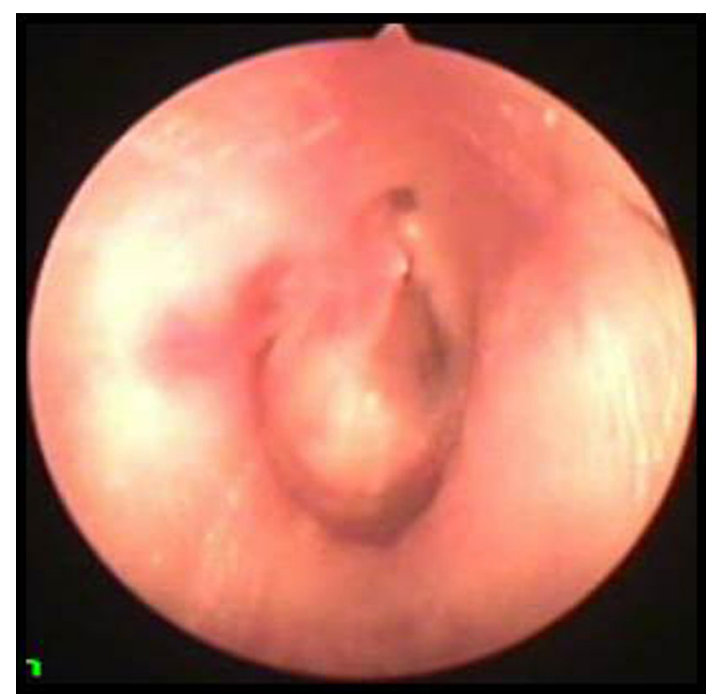

Fig. 171 Year post op view of successful type 1 endoscopic cartilage tympanoplasty

of $14 \mathrm{~cm}$ long and $3 \mathrm{~mm}$ diameter to improve instrument access to middle ear. All procedures performed were recorded for documentation. All procedures were done using our Endoholder attachment exclusively. Advantages and disadvantages of the endoscope holder attachment, if any, during all surgeries were noted and documented precisely.

\section{Results}

The endoscope holder assisted 78 endoscopic type 1 cartilage tympanoplasties have been operated from September 2013 to November 2014 for pars tensa perforations with follow up period ranging from 6 to 20 months, which is definitely a very short follow up to evaluate Tympanoplasty outcomes. This technique, being a developing art and novel concept of two handed technique of endoscopic cartilage tympanoplasty, is thus an early attempt to demonstrate and report the use of our endoscope holder for micro ear surgeries. During the early use of endoscope holder for tympanoplasty we started with small pars tensa perforations and then extended the use of the endoscope holder for large perforations (Table 1).

Endoscope is kept most of the times at the isthmus and if the patient moves a bit, the horizontal arm of the microscope can be easily moved in the upward direction due to its spring action. No middle ear injury is reported in our series.

In our early follow up period ranging from 6 to 20 months in 76 ears operated for endoscopic cartilage tympanoplasty, the graft uptake was seen in ears with one residual perforation and four recurrent perforations giving a success rate of 
Table 1 Gender distribution of perforations

\begin{tabular}{lccc}
\hline Perforations involving quadrants & \multicolumn{3}{l}{ Number of ears } \\
\cline { 2 - 4 } & Male & Female & Total \\
\hline One & 11 & 7 & 18 \\
Two & 14 & 11 & 25 \\
Three & 9 & 8 & 17 \\
All & 13 & 5 & 18 \\
Total & 47 & 31 & 78 \\
\hline
\end{tabular}

$97.206 \%$. Our previous study [3] with sliced tragal cartilage perichondrium graft using microscope gave a success rate of $98.2 \%$. The present study was carried out using the same technique of slicing the cartilage [3] to $0.5 \mathrm{~mm}$ thickness and using it for type 1 tympanoplasty by two handed technique with endoscope of $4 \mathrm{~mm}$ diameter held in our designed endoscope holder. Comparing the results present endoscopic primary cartilage tympanoplasty technique with our published microscopic primary cartilage tympanoplasty outcome [3] the results are equivalent in terms of outcome for short term follow up.

\section{Discussion}

Even though it has been two decades since endoscopy was first used to explore mastoid cavities, the endoscope is used infrequently for surgical management of ear disease [713].

Endoscopic ear surgery allows a minimally invasive approach to the middle ear [14]. The endoscope also allows an elaborate view of the three main elements in tympanoplasty surgery: ear canal, tympanic membrane, and the tympanic ring [15]. In order to augment the applicability and to overcome the disadvantage of single handed technique of endoscopic surgery, the Endoholder has been designed. Initially after designing the holder, its use and applications were tested on dry temporal bone and also for office based oto-endoscopies. Later on its use and applicability was tested for cadaveric temporal bone dissection.

A total of 76 endoscopic cartilage tympanoplasties were operated with endoscope holder by both the authors. During endoscopic cartilage tympanoplasty with the endoscope holder we observed several advantages.

1. Our innovation patent app no 3300/mum/2013 describes.

a. Simple gripping of any operating microscope optical body with mechanically tightening screw to hold Rigid endoscope by means of endoscope holding metallic plate (rigid endoscope too is held in circular opening with mechanical fixing) irrespective of whether microscope functioning or not.

b. By gripping microscope optical system by this way, I use existing all microscope movement in space and apply simply to endoscope movement (which is very important for two hand techniques) [Note: I mean movement of microscope and not function. Hence both systems (endoscope and microscope) are functioning independently].

c. Two handed technique means using both hands of surgeon for operation (at present in endoscopic ENT or other surgeries, surgeon hold endoscope in left hand and uses right hand for doing surgical steps).

d. At present ear surgery is done by using microscope $(90 \%)$ and few surgeons use endoscope $(10 \%)$. presently disadvantage of EES is that one hand is compromised to hold endoscope. But many advantages of endoscopes are surpassing the microscope in ear surgery. Single hand technique will become two hand technique by using our simple innovation (Note: both endoscope and microscope are functioning independently).

e. Why to hold endoscope in metallic plate grip it to microscope?

1. To use both hands for surgery.

2. To apply all movements (not functions) of microscope optical body for endoscope movement.

3. By doing so endoscope can be moved more or less like using left hand which is important for any surgery.

4. We are not using both system(endoscope and microscope) simultaneously.

5. But both system can be used alternatively which give added advantages.

6. Every ENT surgeon owns microscope for ear surgery. Now by using our innovation he will be able to do EES with two hands (at present no body in the world is doing this) with simply fixing one endoscope holding metallic plate.

7. Anything which will be holding endoscope, if fixed to microscope optical body will automatically apply all movements of operating microscope for driving endoscope during surgery (this is the basis of our innovation).

8. Presently EES is in nascent stage and mainly done as single handed technique. By our innovation it will be two handed technique and will open new horizons in many surgical fields including ENT. 
9. Possibly after my demonstration in many congresses, microscope manufacturers may provide inbuilt metallic plate which will be holding endoscope (very simple but nobody thought till this date. Our innovation describe this firstly).

10. Considering the many advantages of two handed technique in endoscopic surgery, our innovation is discovery of new thing which was unknown till this date and very much applicable in many areas.

f. Our simple innovation is "holding rigid endoscope in metallic plate or by any other means and fixing it to operating microscope" the benefits are described in patent.

g. At present endoscopic Ear Nose Throat surgery or any other endoscopic surgery is carried out by holding rigid endoscope in left hand and operating with right hand. Our innovation will allow both hands to be used for surgery as endoscope is held metallic plate and secured to microscope. This allows application of all movements of microscope to endoscope.

h. Alternately means when I want to operate with endoscope I may use endoscope and when microscope, I will use microscope. Note here I am not using both the instruments simultaneously as described in many patents.

i. In simple ways: hold the rigid endoscope and secure it by fixing to microscope optical head for applying all its only mechanical movements to endoscope as your left hand does during holding endoscope for surgery.

j. All present patents describes simultaneous observation through endoscope and microscope. My purpose is different. Just applying microscope movements to rigid endoscope moving.

k. To summarize:

1. Simple innovation of holding endoscope in metallic plate and fixing it to microscope optical body.

2. By doing this idea is to use all microscope mechanical movements not functions for movement of endoscope in space.

3. Instead of developing separate stand to hold endoscope, it can be held as described in our innovation. Thus a very cheap but beneficial alternative.

4. Contemporary single handed technique in EES will be two handed technique.

5. Both systems (endoscope and Microscope) works independently with their own advantages and disadvantage and not remotely related functionally. Only implementation of microscope stand actions for driving endoscope in space mechanically during surgery.

\section{Advantages of Our Endoscope Holder}

1. Both the hands of the surgeon are free for operative intervention which is of paramount importance both for diagnostic and operative procedure.

2. When angled endoscope $\left(30^{\circ}, 45^{\circ}, 70^{\circ}\right)$ is mounted on the endoscope holder allows better visualisation of sinus tympani, facial recess, anterior tympanic cavity and hypotympanum with just rotation of the endoscope within the Holder.

3. The elevation of the tympanomeatal flap and tympanic membrane assessment is much better as the left hand holds the suction cannula continuously (similar to Microscope ear surgery).

4. It can be used effectively without compromising the surgical field.

5. As an effective alternative for documentation and a useful teaching aid.

6. As endoscope is fixed onto the holder, the stability of the endoscope, camera and image on the monitor is ensured throughout.

7. Minimizes the need for assistance.

8. No surgeon fatigue in holding the endoscope as the endoscope is fixed on the endoscope holder (as compared with single handed EES).

9. As the endoholder attachment is mounted on optical system of Microscope Stand, the fine focus of the microscopic stand can be utilized for additional manipulation/advancing into the external auditory canal.

10. Mastoid bandage is not required.

11. Success rate is equivalent in early follow up compared to microscopic technique [3] as the placement of graft is more precise due to the total view of the tympanic membrane (Fig. 17).

12. Fogging of the endoscope is avoided by use of suction cannula.

13. Canalplasty can be done with the drill in the right hand and suction in the left hand.

14. As endoscope and microscope are on same platform, the alternate use of both is possible. This is very much necessary for initial training in EES.

\section{Disadvantages}

1. At times, narrow canal can pose difficulty during endoscopic tympanoplasty, but can be circumvented 
by canalplasty. In this series not a single canalplasty was carried. We use specially designed Karl Storz $14 \mathrm{~cm}$ long, $3 \mathrm{~mm}$ diameter $0^{\circ}$ endoscope in narrow canal.

2. During hemorrhage, the manipulation of the endoscope in and out of the operative field may be time consuming.

3. Possible potential disadvantage is the thermal damage to the middle ear due to endoscope light carrying fibres has been reported previously and measures to avoid excessive temperature elevation in EES has been suggested [16]. This is circumvented by following simple tecniques during the use of our endoholder assisted transcanal two handed endoscopic tympanoplasty.

a. The endoscope is kept at a distance mostly at the isthmus of external auditory canal.

b. Continuous suction is held in left hand during the procedure as suggested in previous study [16].

c. Intermittent irrigation gives the cooling effect to endoscope [16] and added cleaning of the tip of the endoscope which avoids frequent its removal for defogging and cooling as done in single handed EES.

4. Overall cost of the endoscope holder in addition to the routine ear instruments.

5. Care is to be taken to avoid accidental injury due to head movement to the tip of the endoscope.

The learning curve for endoscope holder aided endoscopic tympanoplasty may not as steep as that for routine endoscopic or microscopic surgery. Many experienced with endoscopic surgeries will find the technique easy to master, but surgeons who have limited themselves to an entirely microscopic otology practice are likely to find endoscope holder aided endoscopic surgery more difficult to perform. Despite this, with sufficient training and practice the technique may be adopted by all. It is important to remember that the initial steps of the surgery are the most difficult. Once comfortable with partial use of the endoscope holder movements and manipulation, most surgeons may prefer to use the endoscope holder aided endoscopic surgeries.

\section{Extending the Use of Endoscope Holder for Ear Surgeries}

At present we have demonstrated the early use of our endoscope holder for tympanoplasty. The further use and applicability of endoscope holder needs to be extended to ossiculoplasty and mastoid surgeries. It will be useful to collaborate and associate with other, more experienced surgeons who are more familiar with endoscopic ear, sinus and laryngeal surgeries to predict its consistent progress.

\section{Conclusion}

This Endoholder developed as a modification of microscopic stand, incorporates the endoscope onto the microscope stand and augments the applicability of the endoscope into ENT practice. With little practice, endoscope holder can be used routinely in all ENT surgeries. endoscope holder is a valuable adjunct to conventional endoscopic surgeries. The technique though useful has a different learning curve and training is useful to allow more familiarity when getting started.

\section{Compliance with Ethical Standards}

Conflict of interest Application for Patent in respect of "Rigid Endoscope Holder and Endoscope Fixing Method with Microscope Gripping Attachment" bearing Application No. 3300/MUM/2013 in the name of Dr. Mubarak Khan to Patent controller of India. And "JUSTTACH" is tradename secured for this. Dr Sapna Parab has helped in preparing the manuscript and evaluated endoscopic holder actively during various otolaryngological surgeries independently and along with first author.

Ethical Approval All procedures performed in studies involving human participants were in accordance with the ethical standards of the institutional committee and with the 1964 Helsinki declaration and its later amendments or comparable ethical standards.

Informed Concent Informed consent was obtained from all individual participants included in the study.

\section{References}

1. Tarabichi M (1999) Endoscopic middle ear surgery. Ann Otol Rhinol Laryngol 108(1):39-46

2. Khan MM, Parab SR (2014) Concept, design and development of innovative endoscope holder system for endoscopic otolaryngological surgeries. Indian J Otolaryngol Head Neck Surg. doi: 10.1007/s12070-014-0738-y

3. Khan MM, Parab SR (2011) Primary cartilage tympanoplasty: our technique and results. Am J Otolaryngol 32(5):381-387

4. Khan MM, Parab SR (2013) Reinforcement of sliced tragal cartilage perichondrium composite graft with temporalis fascia in type I tympanoplasty: our techniques and results. J Rhinolaryng Otol 1:57-62

5. Khan MM, Parab SR (2014) Sliced Island tragal cartilage perichondrial composite graft: early results and experience. J Rhinolaryng Otol 2:4-9

6. Khan MM, Parab SR (2015) Comparative study of sliced tragal cartilage and temporalis fascia in type I tympanoplasty. J Laryngol Otol 129(1):16-22

7. Tarabichi M. Endoscopic cholesteatoma, tympanoplasty and middle ear. Open access atlas of otolaryngology, head and neck operative surgery. https://vula.uct.ac.za/access/content/group/ ba5fb1bd-be95-48e5-81be-586fbaeba29d/Endoscopic\%20chole 
steatoma, \%20tympanoplasty\%20and\%20middle $\% 20$ ear $\% 20$ sur gery.pdf

8. Thomassin JM, Korchia D, Doris JM (1993) Endoscopic-guided otosurgery in the prevention of residual cholesteatomas. Laryngoscope 103:939-943

9. Hawke M (1982) Telescopic otoscopy and photography of the tympanic membrane. J Otolaryngol 11:35-39

10. Nomura Y (1982) Effective photography in otolaryngology-head and neck surgery: endoscopic photography of the middle ear. Otolaryngol Head Neck Surg 90:395-398

11. Takahashi H, Honjo I, Fujita A, Kurata K (1990) Transtympanic endoscopic findings in patients with otitis media with effusion. Arch Otolaryngol Head Neck Surg 116:1186-1189
12. Poe DS, Bottrill ID (1994) Comparison of endoscopic and surgical explorations for perilymphatic fistulas. Am $\mathrm{J}$ Otol 15:735-738

13. McKennan KX (1993) Endoscopic 'second look' mastoidoscopy to rule out residual epitympanic/mastoid cholesteatoma. Laryngoscope 103:810-814

14. Pothier D (2013) Introducing endoscopic ear surgery into practice. Otolaryngol Clin N Am 46(2):245-255

15. Tarabichi M (2010) Endoscopic transcanal middle ear surgery. Indian J Otolaryngol Head Neck Surg 62(1):6-24

16. Kozin ED, Lehman A, Carter M et al (2014) Thermal effects of endoscopy in a human temporal bone model: implications for endoscopic ear surgery. Laryngoscope 124(8):E332-E339 